\title{
FACTORES ASOCIADOS AL RENDIMIENTO ACADÉMICO EN ESTUDIANTES UNIVERSITARIOS, UNA REFLEXIÓN DESDE LA CALIDAD DE LA EDUCACIÓN SUPERIOR PÚBLICA
}

\author{
Guiselle María Garbanzo Vargas \\ Directora de la Escuela de Administración Educativa de la \\ Universidad de Costa Rica \\ San José, Costa Rica
}

Recibido 5-VI-2007 • Aceptado 12-VIII-2007

\begin{abstract}
Resumen: Este artículo presenta una revisión de los hallazgos de investigación consignados en la literatura que se señalan como posibles factores asociados al rendimiento académico en estudiantes universitarios, y su vinculación con la calidad de la educación superior pública en general, para lo que se apoya en estudios realizados en España, Colombia, Cuba, México y Costa Rica. Se agrupan en tres categorías: determinantes personales, determinantes sociales $y$ determinantes institucionales que, a su vez, poseen sus propios indicadores. Se hace énfasis en que la búsqueda de la calidad educativa en el sector universitario es un bien deseado por distintos sectores de la sociedad, y por el que luchan las universidades desde diferentes ámbitos, con mayores cuestionamientos en el sector público por la inversión estatal que conlleva. La búsqueda de la calidad implica una revisión integral de la universidad que incluye estudios sobre el rendimiento académico del alumnado, por lo que sus resultados son un insumo importante, pues permiten conocer elementos obstaculizadores y facilitadores del desempeño estudiantil $y$, en consecuencia, permiten favorecer el control de los recursos estatales y la mediación del impacto social.

El análisis de la calidad educativa debe incluir resultados de investigación sobre el rendimiento académico de los estudiantes, pues es de gran utilidad en procesos de toma de decisiones en aras de un sistema educativo más justo.
\end{abstract}

Palabras clave: Rendimiento académico, estudiantes universitarios, educación superior pública, calidad de la educación, educación superior, estudiantes universitarios.

\section{Introducción}

El rendimiento académico del estudiantado universitario constituye un factor imprescindible en el abordaje del tema de la calidad de la educación superior, debido a que es un indicador que permite una aproximación a la realidad educativa. (Díaz, Peio, Arias, Escudero, Rodríguez, Vidal, 2002)

En lo que a calidad de la educación superior se refiere, y partiendo de los distintos cuestionamientos que se le hacen al sector público en cuanto a la relación costo-beneficio social, ha despertado en las autoridades universitarias un interés particular por los resultados académicos de sus estudiantes, cuyo estudio y análisis constituyen herramientas sólidas para construir indicadores que orienten la toma de decisiones en educación superior.

Los estudios del rendimiento académico en la educación superior parecen ser en la coyuntura mundial actual aún más valiosos, debido al dinamismo que experimenta el sector universitario en el marco de una sociedad caracterizada por 


\begin{abstract}
This paper presents a review of the research findings documented in literature about factors associated with academic scores in college students and their relationship with the quality of higher education in general, using as a reference studies carried out in Spain, Colombia, Cuba, Mexico, and Costa Rica. These factors are grouped in three categories: personal determinants, social determinants, and institutional determinants that, at the same time, hold their own indicators. Emphasis is given to the fact that the search of educational quality is a desired good for different sectors of society, and for which universities fight from different fields, with major concerns in the public sector due to the investment involved. The search for quality implies an integral review of the university that includes studies about the academic results of students, because those results are an important input and allow the identification of limiting and enabling elements of academic performance and, consequently, facilitate the control of public resources and the social impact mediation. The analysis of the educational quality must include research results about the academic results of the students since it is of great use in the decision making process aiming for a more fair educational system.
\end{abstract}

Key words: College students, academic results, higher public education, quality of education, el rápido avance del conocimiento, la fluidez en la transmisión de la información y los cambios acelerados en las estructuras sociales. En ese contexto adquiere valor la calificación del capital humano y ello va en estrecha vinculación con los resultados e investigaciones sobre el rendimiento académico de los estudiantes universitarios.

Investigaciones sobre el desempeño estudiantil permiten conocer un gran número de variables que entran en juego en lo que a calidad y equidad de la educación superior pública se refiere, por lo que aportan importantes elementos que repercuten en la gestión y prestigio institucional, sobre todo cuando la inversión estatal es fundamental.

En materia de rendimiento académico en la educación superior, la mayoría de los estudios son cuantitativos, con un marcado interés en el campo económico y son pocas las investigaciones que hacen un abordaje cualitativo del problema. No obstante, sus resultados han permitido identificar factores que favorecen o limitan el desempeño académico. Es así que se procede a especificar los hallazgos de la investigación respecto de componentes asociados al rendimiento académico en estudiantes universitarios que inciden en mayor o menor grado en los resultados y notas que es importante considerar cuando se diseñan estudios de este tipo.

Los indicadores de rendimiento académico que se consideran en este artículo se sustentan en una revisión teórica, y en su mayoría han sido usados en distintos estudios en la educación superior, en diferentes momentos, diferentes universidades y por diferentes investigadores, y se presentan con una perspectiva enmarcada hacia la calidad de la educación.

\section{Contextualización}

La educación superior en América Latina experimentó, en la década de 1990, un marcado interés por la calidad educativa, al reconocer en ella la principal 
herramienta para responder a las exigencias y demandas educativas en un contexto marcado por desafíos propios del proceso de la globalización. Al respecto, Beck (1999) afirma que en el mundo globalizado se le atribuye un lugar especial al conocimiento, aduciendo que se tenderá a valorar de manera creciente el avance teórico y la innovación tecnológica, por lo que la inversión en la formación y en la investigación se vuelve indispensable para la producción y reproducción del sistema social y económico.

En el marco de la globalización, la educación superior en América Latina enfrenta la necesidad de una convergencia y articulación de sus sistemas, así como la construcción y consolidación del espacio latinoamericano de educación superior, que son importantes desafíos ante la fragmentación, diversificación institucional y disparidad que se presenta en la educación superior desde la década de 1990.

El proceso de diversificación de la educación superior en la región desde 1990 hasta hoy, tanto en su organización como en cuanto a la calidad, ha sido muy diferente al que prevaleció en el siglo XX, hasta la década de 1980, donde la educación superior había sido principalmente estatal, acompañada de una fuerte autonomía institucional y académica. Esta tendencia se inició con el movimiento de la reforma universitaria de 1918, en la Universidad Nacional de Córdoba. (Lamarra, 2004)

En la década de 1990, en el contexto de la globalización, emergieron estrategias y políticas neoliberales con una marcada orientación al reemplazo de las políticas protectoras, por la visión del mercado y la privatización de la educación superior pública. Este cambio en las políticas estatales se materializó en fuertes restricciones del financiamiento público a las universidades, y cuestionamientos por la relación entre el costo estatal y los resultados en cuanto al número y la calidad de los graduados

Otra tendencia en la era de la globalización es el aumento en la demanda por educación superior. En respuesta a esta situación, se crean diferentes instituciones de educación superior; muchas de ellas de carácter privado, lo que ha ido en detrimento de la calidad y la pertinencia social (Fernández, 2004). Para el caso América Latina, en la segunda mitad del siglo XX, el número de instituciones y la matrícula anual experimentaron un incremento sostenido y en la década de 1990 creció un 6\% (8\% sector privado y 2,5 sector público) tal y como se ilustra en los siguientes cuadros.

\section{Cuadro $\mathrm{N}^{\circ} 1$}

Instituciones universitarias en América Latina

\begin{tabular}{cc}
\hline Año & Instituciones \\
\hline 1950 & 75 \\
1975 & 330 \\
1985 & 450 \\
1995 & 812 (319 públicas \\
& y 493 privadas) \\
2003 & Más de 1.500 \\
\hline
\end{tabular}

Fuente: Tomado de (Lamarra, 2004, p. 2).

Cuadro $\mathrm{N}^{\circ} 2$

Número de estudiantes de educación superior en América Latina

\begin{tabular}{cc}
\hline Año & Instituciones \\
\hline 1950 & 267.000 \\
1970 & 1.640 .000 \\
1980 & 4.930 .000 \\
1990 & 7.350 .000 \\
2000 & 2.000 .000 aproximadamente \\
\hline
\end{tabular}

Fuente: Tomado de (Lamarra, 2004, p. 2).

En el marco de la explosión cuantitativa y la pérdida de calidad de la educación superior, creció el interés por estudiar los factores asociados al rendimiento académico en estudiantes universitarios, a fin de ofrecer herramientas de trabajo a futuras investigaciones en este campo, desde un enfoque más integral sobre el desempeño estudiantil. 


\section{Rendimiento académico en estudiantes universitarios. Conceptualización}

El rendimiento académico de los estudiantes universitarios constituye un factor imprescindible y fundamental para la valoración de la calidad educativa en la enseñanza superior ${ }^{1}$.

El rendimiento académico es la suma de diferentes y complejos factores que actúan en la persona que aprende, y ha sido definido con un valor atribuido al logro del estudiante en las tareas académicas. Se mide mediante las calificaciones obtenidas, con una valoración cuantitativa, cuyos resultados muestran las materias ganadas o perdidas, la deserción y el grado de éxito académico Pérez, Ramón, Sánchez (2000), Vélez Van, Roa (2005).

Las notas obtenidas, como un indicador que certifica el logro alcanzado, son un indicador preciso y accesible para valorar el rendimiento académico, si se asume que las notas reflejan los logros académicos en los diferentes componentes del aprendizaje, que incluyen aspectos personales, académicos y sociales Rodríguez, Fita, Torrado (2004).

Cada universidad determina criterios evaluativos propios, para obtener un promedio ponderado (valoración) de las materias que cursa el estudiante, donde se toman en cuenta elementos como la cantidad de materias, el número de créditos y el valor obtenido en cada una de ellas, que generalmente se denomina "nota de aprovechamiento". En las calificaciones como medida de los resultados de enseñanza hay que tomar en cuenta que son producto de condicionantes tanto de tipo personal del estudiante, como didácticas del docente, contextuales e institucionales, y que todos estos factores median el resultado académico final.

Por sus características, hay consenso en la comunidad de especialistas de lo difícil que resulta identificar el rendimiento académico en la educación superior, debido a que es problemático y confuso identificar el rendimiento académico con las notas. DeMiguel (2001 citado en Rodríguez, Fita, Torrado (2004) advierte al respecto que se debe diferenciar entre el rendimiento académico inmediato -refiriéndose a las notas-, y el mediato -refiriéndose a los logros personales y profesionales-.

Latiesa (1992), citado en Rodríguez, Fita, Torrado (2004), hace una valoración más amplia del rendimiento académico, pues lo evalúa en relación con el éxito, retraso y abandono, y en un sentido más estricto por medio de las notas. La valoración del rendimiento académico no conduce a otra cosa que a la relación entre lo que se aprende y lo que se logra desde el punto de vista del aprendizaje, y se valora con una nota, cuyo resultado se desprende de la sumatoria de la nota de aprovechamiento del estudiante en las diferentes actividades académicas, a las que se sometió en un ciclo académico determinado.

Las observaciones sobre la influencia de los sistemas educativos en el rendimiento académico suelen ser arriesgadas. Se critica por la falta de adecuación contextual de los diferentes estudios, argumentando que el rendimiento no solo tiene efectos diferenciales según un contexto determinado, sino que hay que tomar en cuenta los criterios de evaluación con que se presentan los estudios, ya que su metodología condiciona los resultados. De ahí la dificultad de realizar generalizaciones en torno a un tema tan complejo en contextos disímiles.

Estudios llevados a cabo por Vélez Van, Roa (2005), con estudiantes universitarios en Colombia; Carrión (2002) en Cuba; Valle, González, Núñez, Martínez, Pineñor, (1999) en la Universidad de Coruña, en España y Montero, Villalobos, en la Universidad de Costa Rica, se ha abordado el tema del rendimiento académico a partir de variables cognitivas, emocionales y socioeconómicas, entre las que se incluyen resultados de la educación 
secundaria, pruebas del Estado, el examen de ingreso a la universidad, las pruebas de aptitudes intelectuales, factores psicosociales (consumo de alcohol y otro tipo de sustancias), rasgos de personalidad y factores relacionados con el estado emocional hacia el estudio, calificaciones obtenidas, composición familiar, el interés vocacional, los hábitos de estudio y el nivel académico de los padres.

Conocer los diferentes factores que inciden en el rendimiento académico en el campo de la educación superior de una manera más integral, permite obtener resultados tanto cualitativos como cuantitativos para propiciar un enfoque más completo en la toma decisiones para mejorar los niveles de pertinencia, equidad y calidad educativa. La determinación de indicadores de índole cuantitativa y cualitativa no implica que los factores asociados al rendimiento académico que se tomen en cuenta son exclusivos del campo universitario. Por su complejidad, algunos de ellos son fácilmente adaptables a otras realidades, lo que muestra su capacidad explicativa y analítica en relación con el éxito académico en cualquier sector educativo, independientemente de si se trata de instituciones públicas o privadas.

\section{Factores que intervienen en el rendimiento académico}

El rendimiento académico, por ser multicausal, envuelve una enorme capacidad explicativa de los distintos factores y espacios temporales que intervienen en el proceso de aprendizaje. Existen diferentes aspectos que se asocian al rendimiento académico, entre los que intervienen componentes tanto internos como externos al individuo. Pueden ser de orden social, cognitivo y emocional, que se clasifican en tres categorías: determinantes personales, determinantes sociales y determinantes institucionales, que presentan subcategorías o indicadores.
Para que los estudios de rendimiento académico sean útiles, es importante identificar el tipo de influencia de los factores asociados al éxito o al fracaso del estudiantado; es decir, de los niveles de influencia entre las variables por considerar para determinar factores causales y mediaciones que determinan las relaciones entre las distintas categorías de variables personales, sociales e institucionales. Estas variables, además de ofrecer información de carácter estructural y objetivo, toman en cuenta la percepción del estudiante respecto de factores asociados al rendimiento académico y a su posible impacto en los resultados académicos. La mayoría de estudios sobre rendimiento académico se basan en una aproximación metodológica de tipo predictivo, donde se utilizan modelos de regresión múltiple, pocas veces complementados con modelos explicativos que favorecen un análisis más integral de los factores asociados al rendimiento académico, por lo que es útil describir las características de los determinantes mencionados (Castejón, Pérez, 1998).

\subsection{Determinantes personales}

En los determinantes personales se incluyen aquellos factores de índole personal, cuyas interrelaciones se pueden producir en función de variables subjetivas, sociales e institucionales. La siguiente figura muestra algunos factores asociados al rendimiento académico de índole personal, agrupados en la categoría denominada determinantes personales, que incluye diversas competencias.

La competencia cognitiva se define como la autoevaluación de la propia capacidad del individuo para cumplir una determinada tarea cognitiva, su percepción sobre su capacidad y habilidades intelectuales. Está relacionada con la influencia ejercida en el entorno familiar e incide en distintas variables que se asocian con el éxito académico tales como: la persistencia, 
el deseo del éxito, expectativas académicas del individuo y la motivación. El afecto de los padres hacia el estudiante se asocia con el establecimiento de una alta competencia académica percibida y con la motivación hacia el cumplimiento académico. (Pelegrina, García y Casanova, 2002)

Figura N. ${ }^{\circ} 1$

Factores asociados al rendimiento académico en estudiantes universitarios Determinantes Personales

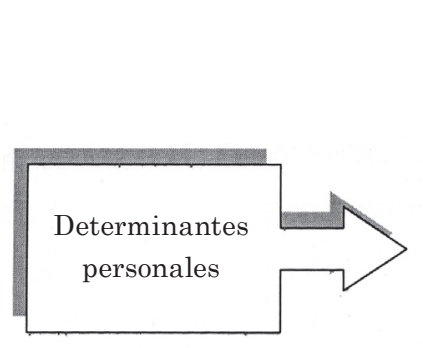

Competencia cognitiva

Motivación

Condiciones cognitivas

Autoconcepto académico

Autoeficacia percibida

Bienestar psicológico

Satisfacción y abandono con respecto a los estudios

Asistencia a clases

Inteligencia

Aptitudes

Sexo

Formación académica previa a la Universidad

Nota de acceso a la universidad

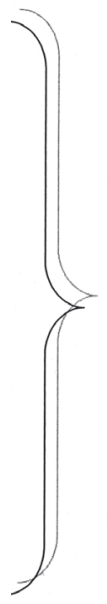

Salonava, M., Cifre, Eva, Grau, R., Martínez, M. Isabel (2005), dentro del marco de la teoría social cognitiva, hacen referencia a la competencia cognitiva como aquellas creencias en las propias capacidades para organizar y ejecutar lo necesario para obtener el nivel de logro deseado, o autoeficacia, entendida como capacidad o habilidad intrínseca del individuo para el logro de las metas, que es una característica trascendental en el desempeño académico. Esta característica se cimienta en la repetición de experiencias de éxito que el individuo haya alcanzado, observando el logro en los demás, críticas verbales positivas, y los estados emocionales y somáticos. Es un hecho que cada individuo tiene su propia interpretación de sus estados emocionales y somáticos; interpretación que repercute en la construcción de la competencia cognitiva, en la medida que los estados emocionales y somáticos sean positivos o negativos, incidirán en la construcción de la competencia cognitiva.
Estudios llevados a cabo con estudiantes universitarios pusieron en evidencia que aquellos estudiantes con creencias de autoeficacia académica positiva, se asocian con resultados de éxito académico. (Oliver, 2000)

La motivación es otro determinante que se subdivide en distintas facetas: motivación intrínseca, extrínseca, atribuciones causales y percepciones de control.

a) La motivación académica intrínseca: está ampliamente demostrado que la orientación motivacional del estudiante juega un papel significativo en el desempeño académico. Algunos autores como Salonava, Martínez, Bresó, Llorens Gumbau S., Gumbau Grau R. (2005, p. 173), se refieren a este campo como el engagement, definido como "un estado psicológico relacionado con los estudios que es positivo y significativo" El engagement es caracterizado por vigor, dedicación y abosorción. 
El vigor se caracteriza por altos niveles de energía y resistencia mental, mientras se trabaja, el deseo de invertir esfuerzo en el trabajo que se está realizando incluso cuando aparecen dificultades en el camino... (Salonava, Cifre, Grau, Martínez, 2005, p. 163)

Por su parte, la dedicación conlleva una alta implicación en las tareas, por lo que se experimenta entusiasmo, inspiración, orgullo y reto por lo que se hace, y la absorción ocurre cuando se experimenta un alto nivel de concentración en la labor.

Estas condiciones se aprecian en aquellos estudiantes para quienes el estudio significa más que una tarea un disfrute por el saber, pueden pasar largas horas desarrollando actividades académicas con una gran disposición hacia lo que hacen, debido a que tienen la sensación de que el tiempo pasa demasiado rápido y muestran una alta capacidad de compromiso y concentración académica. Por lo general, estas personas manifiestan sentir felicidad al realizar las tareas académicas, pues el estudio es un disfrute. Las investigaciones sobre el engagement han mostrado la influencia positiva en el funcionamiento personal y social en distintos contextos, como lo es el campo académico. Las creencias de eficacia ofrecen al individuo herramientas automotivadoras que le movilizan a hacer el esfuerzo que exige la búsqueda de las metas y la persistencia para alcanzar lo que se desea lograr ${ }^{2}$.

La motivación la determinan las diferentes interpretaciones y valoraciones que un individuo construye sobre sus resultados académicos; según Weiner, citado por Valle, González, Núñez, Martínez, Pineñor (1999), una secuencia motivacional parte de un resultado determinado, dando una reacción afectiva inmediata en el individuo.

Dentro de la motivación en lo que a aspectos de resultados académicos se refiere, una variable que ha demostrado su importancia son las metas académi- cas del estudiante. Se subdividen en metas de aprendizaje y metas de rendimiento académico y están fuertemente vinculadas a la motivación intrínseca y extrínseca. En referencia a este tema es que Valle, González, Núñez, Martínez, Pineñor (1999) afirman que los motivos, razones e intenciones que orientan el comportamiento académico del estudiante determinan en gran medida el tipo de recursos cognitivos que emplea al enfrentarse con los procesos educativos, y se le conoce como "condiciones cognitivas". Por su parte, Castejón, Pérez (1998) señala la existencia de dos móviles fundamentales que orientan el comportamiento humano: la consecución del éxito y la evitación del fracaso. Al respecto, Weiner (1986), citado por el mismo autor, indica que el comportamiento motivacional está en función de las expectativas por alcanzar la meta y el valor de incentivo que se le asigna.

b) La motivación extrínseca: se relaciona con aquellos factores externos al estudiante, cuya interacción con los determinantes personales da como resultado un estado de motivación. Dentro de los elementos externos al individuo que pueden interactuar con los determinantes personales, se encuentran aspectos como el tipo de universidad, los servicios que ofrece la institución, el compañerismo, el ambiente académico, la formación del docente y condiciones económicas entre otras. La interacción de estos factores externos puede afectar la motivación del estudiante para bien o para mal, por lo que se asocia con una repercusión importante en los resultados académicos.

c) Las atribuciones causales: se refieren a la percepción que tiene el individuo sobre el desarrollo de la inteligencia $\mathrm{y}$, en consecuencia, de los resultados académicos, en el sentido de si se atribuye que la inteligencia se desarrolla con el esfuerzo o es casual; es decir, si los resultados académicos son consecuencia del nivel de esfuerzo 
del estudiante, de su capacidad, del apoyo recibido o un asunto de suerte. Se ha demostrado que asumir que los resultados académicos se deben a la propia capacidad y esfuerzo, ello influye en el logro de buenos resultados académicos ${ }^{3}$.

d) Las percepciones de control: constituyen en la percepción del estudiante sobre el grado de control que se ejerce sobre su desempeño académico y pueden ser cognitivas, sociales y físicas. Desde el punto de vista cognitivo, Pelegrina, Linares y Casanova (2002), establecen tres fuentes de control:

- Interno: cuando el resultado depende del propio estudiante, y tiene fuerte relación con la motivación del estudiante hacia las tareas académicas.

- Control con los otros: cuando el resultado depende de otras personas, que ejercen control sobre los resultados que se esperan del alumno, no se lucha únicamente por lo que el alumno desea alcanzar, sino por lo que otros desean que el alumno logre, se da una relación asimétrica en lo que a logro se refiere entre el estudiante y terceras personas.

- Desconocido: cuando no se tiene idea de quién depende el resultado.

Un estudio realizado por Pérez, Ramón, Sánchez (2000) con estudiantes universitarios destaca que la falta de motivación de los alumnos se refleja en aspectos como ausencia a clases, bajos resultados académicos, incremento de la repitencia y en el abandono de sus estudios.

Las condiciones cognitivas son estrategias de aprendizajes que el estudiante lleva a cabo relacionadas con la selección, organización, y elaboración de los diferentes aprendizajes. Se definen como condiciones cognitivas del aprendizaje significativo. La orientación motivacional da pie a la adopción de metas, que determinan en gran medida las estrategias de aprendizaje que el estudiante emplea y repercuten en su rendimiento académico. La percepción que el estudiante construya sobre factores como la evaluación, el tipo de materia, la complejidad de la materia y el estilo de enseñanza, influyen en las estrategias de aprendizaje ${ }^{4}$. El uso de mapas conceptuales, hábitos de estudio, horas asignadas al estudio, y las prácticas académicas son algunas estrategias de aprendizaje utilizadas por los estudiantes.

El autoconcepto académico está fuertemente vinculado con la motivación del estudiante y sus resultados académicos. Se define como el conjunto de percepciones y creencias que una persona posee sobre sí misma, es así como la mayoría de variables personales que orientan la motivación parten de las creencias y percepciones que el individuo tiene sobre aspectos cognitivos.

La capacidad percibida por parte del estudiante, el rendimiento académico previo y creer que la inteligencia se desarrolla a partir del esfuerzo académico, contribuyen a mejorar un autoconcepto académico positivo. No en vano en las últimas décadas se ha incorporado el autoconcepto académico como una variable motivacional. En un estudio realizado por Valle, González, Núñez, Martínez, Pineñor (1999) con estudiantes universitarios en la Universidad de Coruña, España, se tuvo como eje la variable motivacional, con base en dos de las perspectivas teóricas que mayor relevancia han tenido en los últimos años: los procesos de atribución causal y el enfoque de la motivación centrado en las metas académicas. Paralelamente a estas perspectivas teóricas, se introdujo la variable autoconcepto académico del estudiante universitario por considerarse fundamental en los resultados académicos.

Los resultados del estudio mostraron que la capacidad, el esfuerzo, la suerte y la dificultad de la tarea académica son los factores causales a los que los estudiantes 
acuden con más frecuencia para justificar sus resultados académicos. Según la causa que el estudiante atribuya, así va a incidir sobre el autoconcepto, su confianza en sus capacidades y en las conductas futuras de logro académico. El rendimiento académico previo influye sobre el autoconcepto académico y esta relación, a su vez, repercute en los resultados académicos actuales ${ }^{5}$.

Estudios citados por Castejón, Pérez (1998), prueban la existencia de una relación entre el autoconcepto académico y el rendimiento académico en interacción con otras variables como la clase social.

En la autoeficacia percibida se dan casos de estudiantes que por distintas razones carecen de autoeficacia. Esta condición se presenta cuando hay ausencia de un estado de motivación intrínseca que permita al estudiante cumplir con un desempeño académico aceptable. Se asocia con estados de agotamiento, desinterés y falta de proyección con sus estudios, y es conocido como burnout, que es la fatiga o la sensación de estar "quemado" por las actividades académicas. La motivación y el compromiso de los estudiantes con el logro académico son fundamentales en sus resultados. (Pérez, Ramón, Sánchez, 2000)

Bienestar psicológico: estudios como los de Oliver, 2000, señalan una relación importante entre bienestar psicológico y rendimiento académico. Estudiantes con mejor rendimiento académico muestran menos burnout y más autoeficacia, satisfacción y felicidad asociadas con el estudio y es común en aquellos estudiantes que no proyectan abandonar los estudios. Se ha encontrado que cuanto mayor rendimiento académico haya habido en el pasado, mayor será el bienestar psicológico en el futuro, y este, a su vez, incidirá en un mayor rendimiento académico y viceversa. Lo mismo sucede con las creencias de eficacia académica y las relaciones entre éxito académico.
La satisfacción hace referencia al bienestar del estudiante en relación con sus estudios, e implica una actitud positiva hacia la universidad y la carrera. El abandono se refiere a las posibilidades que el estudiante considera de retirarse de la universidad, de la carrera o del ciclo lectivo. "La superación de retos y la consecución de objetivos aumenta la autestima, la autoeficacia y en general produce satisfacción”. (Salonava, Cifre, Grau, Martínez, 2005, p. 171).

La satisfacción personal tiene un papel importante en el desempeño académico, como mostró el estudio realizado por Vélez, Roa (2005) con estudiantes universitarios en la Universidad del Rosario EB Bogotá, Colombia, donde la mayoría de estudiantes que estaba satisfecha con su carrera y universidad, y alejados de presiones por parte de sus padres presentaron resultados positivos en el rendimiento académico.

La asistencia a clases se refiere a la presencia del alumno en las lecciones. En la investigación realizada por Pérez, Ramón, Sánchez (2000) con estudiantes universitarios, se encontró que la motivación está asociada a la asistencia a clases, y que la ausencia a las lecciones se relaciona con problemas de repetición y abandono a los estudios. Cuanta mayor asistencia, mejor calificación; la asistencia es una de las variables más significativas que influye en el rendimiento académico del alumno. Conclusiones similares las encontró Montero y Villalobos (2004) en estudio realizado con universitarios de la universidad de Costa Rica, en la que se envidenció que los estudiantes y las estudiantes que asisten a clases regularmente siempre o casi siempre tienen en promedio 0,26 puntos más que aquellos estudiantes que no lo hacen en forma regular.

La inteligencia es una de las variables más estudiadas dentro de los 
determinantes de índole personal. Incluye pruebas de comprensión verbal y razonamiento matemático (pruebas psicométricas). La inteligencia es un buen predictor de los resultados académicos, que sobresale en el rendimiento académico, lo cual produce una relación significativa entre inteligencia y rendimiento académico; sin embargo, los coeficientes de correlación son moderados, lo que podría asociarse con la influencia recibida de variables como las sociales e institucionales. (Castejón, Pérez, 1998)

En lo que a inteligencia se refiere, es importante identificar el tipo de inteligencia que se desee valorar como la emocional o la social por ejemplo, y seleccionar adecuadamente sus metodologías evaluativas. Sobre la inteligencia emocional, en estudio realizado por Montero y Villalobos (2004) con estudiantes universitarios en Costa Rica, se indica que el puntaje en la Escala de Inteligencia Emocional en contextos académicos y el promedio de admisión a la universidad presentan importantes niveles de asociación.

Aptitudes: al igual que la inteligencia, las aptitudes son variables comúnmente estudiadas dentro de los determinantes de índole personal. Se asocian a habilidades para realizar determinadas tareas por parte del estudiante, mediante diferentes pruebas Castejón, Pérez, 1998)

El sexo: no se puede afirmar del todo una relación directa con el rendimiento académico y el sexo; sin embargo, hay estudios que le dan a la mujer una ligera tendencia al rendimiento superior que a los hombres (Rodríguez, S., Fita, S., Torrado, M. (2004), González, F. A. , 1996).

Estudio realizado por Montero y Villalobos (2004) en la Universidad de Costa Rica encontró relación significativa entre el sexo y el promedio ponderado del estudiante, lo cual viene a contribuir, a criterio de las investigadoras, en hallazgos de investigación similares en las recientes tendencias internacionales orientadas a la superioridad de las jóvenes en indicadores de rendimiento académico.

Formación académica previa a la universidad: diferentes estudios explican que el rendimiento académico previo a la universidad es un claro indicador del éxito académico en los estudios universitarios. Se han estudiado en esta variable indicadores como las calificaciones obtenidas en la educación secundaria, incluyendo pruebas de bachillerato. El rendimiento académico previo a la universidad constituye uno de los indicadores con mayor capacidad predictiva en el rendimiento académico en estudiantes universitarios y tiene mucho que ver la calidad educativa de la institución de la que proviene el estudiante $^{6}$.

Nota de acceso a la Universidad: estudios realizados en la enseñanza superior asociados al rendimiento académico enfatizan el valor de la nota obtenida en las pruebas de admisión a la universidad como un predictor de los más importantes en el rendimiento académico, junto con los rendimientos académicos previos a la universidad. Esta subcategoría se encuentra traslapada, a su vez, con los determinantes institucionales, en el sentido de que el puntaje obtenido y con el que se logra el ingreso a la universidad está determinado por políticas universitarias en lo que a cupos se refieren.

Sobre este tema, Toca y Tourón (1989, p. 32) comprobaron que "aquellos países que practican un proceso de selección pormenorizado y completo, en cuanto a los requisitos de entrada presentan un bajo grado de fracaso entre sus estudiantes”. Carrión (2002) coincide con los autores mencionados, al afirmar que el puntaje en las pruebas de admisión a la universidad son fundamentales, e indica que los exámenes de ingreso desempeñan un papel relevante en esta materia.

Montero y Villalobos (2004) también coinciden en que el promedio de admisión a la Universidad es estadísticamente significativo y de importancia práctica como 
predictor del promedio ponderado del estudiante, en el estudio en que estas investigadoras realizaron el promedio de admisión a la Universidad presenta uno de los coeficientes beta más elevados en el grupo de variables independientes.

4.2. Los determinantes sociales. Son aquellos factores asociados al rendimiento académico de índole social que interactúan con la vida académica del estudiante, cuyas interrelaciones se pueden producir entre sí y entre variables personales e institucionales. La siguiente figura ilustra los factores asociados al rendimiento académico de índole social, agrupados en la categoría denominada determinantes sociales.

Figura N. ${ }^{\circ} 2$

Factores asociados al rendimiento académico en estudiantes universitarios Determinantes Sociales

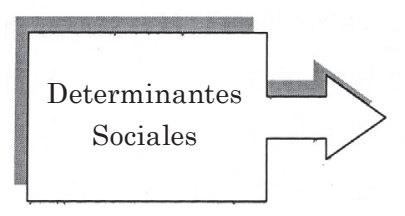

Diferencias sociales

Entorno familiar

Nivel educativo de los progenitores o adultos responsables del estudiante

Nivel educativo de la madre

Contexto socioeconómico

Variables demográficas
Diferencias sociales. Está ampliamente demostrado que las desigualdades sociales y culturales condicionan los resultados educativos. Marchesi (2000) cita un informe de la OCDE-CERI de 1995, donde señala que factores como la pobreza y la falta de apoyo social están relacionados con el fracaso académico; advierte que, sin embargo, no existe una correspondencia estricta entre las desigualdades sociales y las educativas, aduciendo que hay otros factores como la familia, el funcionamiento del sistema educativo y la misma institución que pueden incidir en forma positiva o negativa en lo que a desigualdad educativa se refiere.

El entorno familiar ocupa un lugar importante. Se entiende por entorno familiar un conjunto de interacciones propias de la convivencia familiar, que afectan el desarrollo del individuo, manifestándose también en la vida académica. La influencia del padre y la madre, o del adulto responsable del estudiante, influye significativamente en la vida académica. Un ambiente familiar propicio, marcado por el compromiso, incide en un adecuado desempeño académico, así como una convivencia familiar democrática entre padres e hijos. Se asocia la convivencia familiar democrática con un mejor desempeño académico, que se plasma en variables como motivación, percepción de competencia y atribución de éxito académico; no sucede lo mismo en estudiantes marcados por ambientes familiares autoritarios e indiferentes, de parte de sus padres.

Los comportamientos de los padres median en los resultados académicos de los estudiantes. Un ambiente familiar que estimule el placer por las tareas académicas, la curiosidad por el saber, la persistencia hacia el logro académico se relaciona con resultados académicos buenos (Pelegrina, Linares, Casanova, 2001). Las expectativas del rendimiento académico del estudiante 
por parte de los padres y el control que ejercen sobre ellos tiene repercusión en el rendimiento académico de los estudiantes.

Entornos familiares marcados por la violencia familiar han indicado su relación con resultados académicos insuficientes. Datos de este tipo obtenidos en diferentes investigaciones muestran que la presencia de violencia familiar es un factor asociado al fracaso académico. (Vélez, Roa, 2005)

Padres y madres que se caracterizan por conductas democráticas, influyen en la motivación académica en forma positiva, ya que despiertan actitudes efectivas por el estudio, donde la persistencia y la inquietud por el saber son estimulados, no ocurre lo mismo en ambientes familiares cargados de conductas arbitrarias e indiferentes. El apoyo familiar representa un primer paso hacia el logro en el desempeño académico.

Otro elemento no menos importante en el entorno familiar que tiene que ver con el rendimiento académico, se refiere al nivel educativo del padre y de la madre. El nivel educativo de la madre se abordará por separado dada la relevancia del tema y vale destacar que cuanto mayor es el nivel educativo de los progenitores y en especial de la madre, mayor incidencia positiva hay sobre el rendimiento académico en general. (Castejón y Pérez, 1998)

En lo que a entorno familiar se refiere, se afirma que:

Los recursos familiares, su nivel de estudios, los hábitos de trabajo, la orientación y el apoyo académico, las actividades culturales que se realizan, los libros que se leen, la estimulación para explorar y discutir ideas y acontecimientos y las expectativas sobre el nivel de estudios que pueden alcanzar los hijos, son factores que tienen una influencia muy importante en al educación de los hijos. (Marchesi, 2000, p. 2)

En relación con el nivel educativo de los progenitores o adultos responsables del estudiante, los hallazgos de la investigación manifiestan que el nivel educativo de los progenitores influye significativamente en los resultados académicos. El estudio de Vélez y Roa (2005) con estudiantes universitarios en Bogotá, encontró que el hecho de que no se realicen estudios superiores por parte de los adultos de quien dependen económicamente los estudiantes, se asocia con el fracaso académico. Esta correlación ha sido fuertemente analizada en muchas investigaciones de alto nivel, entre las que se distinguen las efectuadas por organismos internacionales como la CEPAL (Comisión Económica para América Latina y el Caribe).

Sobre este tema, el estudio llevado a cabo por Montero y Villalobos (2004) en la Universidad de Costa Rica no encontró los mismos resultados sobre la relación entre el nivel educativo de los progenitores y el rendimiento académico de los estudiantes universitarios.

Cohen (2002) hace referencia a estudios del Banco Mundial, en los que se demuestra que el $60 \%$ de los resultados académicos se explican por factores fuera del entorno educativo, donde el clima educativo del hogar y los años de estudios de los adultos son los factores de mayor repercusión en los resultados académicos

Castejón y Pérez (1998) plantea que la estimulación educativa de parte de padres con mayor nivel sociocultural es la responsable de las diferencias de los resultados académicos en estudiantes de diferentes niveles económicos.

Nivel educativo de la madre: si bien es cierto anteriormente se hizo referencia al nivel educativo de los progenitores que incluye a la madre, se ha separado con especial interés el nivel educativo de la madre por ser una variable de relevancia en esta temática, como lo indican (Marchesi, 2000; Castejón y Pérez, 1998; quienes consideran:

- cuanto mayor sea el nivel académico de la madre, mayor percepción de apoyo hacia sus estudios tienen los hijos e hijas, lo cual suele reflejarse en el rendimiento académico alcanzado. 
Cuanto mayor nivel educativo tenga la madre, mayores exigencias académicas les plantea a sus hijos, un poco sustentado en la creencia que ellas mismas poseen de que cuanto más asciendan sus hijos académicamente, mayores posibilidades de éxito futuro tendrán.

Este tipo de madres les dan mayor importancia a los deberes académicos, como un medio de incrementar el rendimiento académico de sus hijos, muy distinto a lo que suele suceder con aquellas madres con ausencia o menores niveles educativos.

El nivel académico de la madre en estudios asociados al rendimiento académico se presenta como una variable explicativa, aunque sea en forma indirecta, y no el nivel educativo del padre tal y como lo confirma Castejón y Pérez (1998) en estudio realizado, donde se atribuye como un asunto de tipo sociocultural, aduciendo el hecho de que los padres suelen delegar en las madres la educación de sus hijos, independientemente del nivel socioeconómico, educativo y cultural de la familia.

$\mathrm{Al}$ incremento del nivel educativo de la madre, éste favorece un fortalecimiento hacia lo educativo. Mujeres con mejores niveles educativos son madres que tienden a tener una actitud positiva hacia el estudio de sus hijos, más preocupadas por el desempeño de ellos y con una mayor orientación hacia la importancia de la continuación de los estudios hasta su titulación. (Marchesi, 2000)

Capital cultural: hace referencia al conjunto de relaciones entre el ambiente familiar, sus recursos didácticos como acceso al Internet, a literatura, relaciones familiares marcadas por discusiones que propician el saber, por la búsqueda constante de experiencias que enriquezcan un ambiente educativo; todo este capital cultural contribuye a resultados académicos positivos.
Con respecto al acceso a Internet, este se ha convertido en una poderosa causa de desigualdad; las personas que tengan más facilidades de este tipo, de hecho están mejor preparadas para adaptarse a la sociedad del conocimiento, pues tiene un valor agregado importante que es el ampliar la cultura entre muchos, sucediendo todo lo contrario en condiciones contradictorias. A manera de ejemplo sobre este tema y según datos del Informe sobre Desarrollo Humano de 1998 , el $20 \%$ de la población más rica acapara el 93,3\% del uso de Internet. (Marchesi, 2000)

Contexto socioeconómico: numerosos estudios han permitido establecer correlaciones entre el aprendizaje y el contexto socioeconómico, atribuyendo a causales económicas el éxito o fracaso académico, sin embargo, en este punto hay que tener cuidado, ya que sin bien es cierto el contexto socioeconómico afecta el nivel de calidad educativa, pero que de ningún modo lo determinan si atienden a otras causales, se requerirán estudios específicos para conocer otro tipo de correlaciones, que permitan hacer con exactitud esta determinación causal (Seibold, 2003).

Cohen (2002) hace referencia a un estudio del Banco Mundial en 1995, donde se demostró que entre un $40 \%$ y $50 \%$ de los resultados académicos está fuertemente asociado al impacto de las características del contexto socioeconómico y familiar. También menciona aspectos relacionados con la infraestructura física de la vivienda, destacando características de hacinamiento. Por su parte, Castejón y Pérez (1998) hace referencia a lo usual que es encontrar investigaciones que apoyan la tesis de que en la medida que se asciende en la escala social (nivel económico), los resultados académicos son mejores.

Estudio realizado por Montero y Villalobos (2004) con estudiantes universitarios en la Universidad de Costa Rica 
indica que un resultado generalmente aceptable es la existencia de una asociación significativa entre el nivel socioeconómico del estudiante y su desempeño académico. A su vez, hacen ver la compleja realidad de que una institución educativa pueda incidir para cambiar la condición socioeconómica del estudiante de bajos niveles socioeconómicos; sin embargo, estudios de este tipo son importantes desde el punto de vista científico, que ofrecen un modelo explicativo del fenómeno. Ahora bien, el estudio específicamente realizado por Montero y Villalobos (2004) en el caso del índice socioeconómico del estudiante, a pesar de que el valor de beta no llegó a 0,10 , sí se demostró una relación estadísticamente significativa con la variable dependiente; sorprendiendo que la dirección de la asociación es inversa. Por cada punto que aumenta el índice socioeconómico, el promedio ponderado disminuye. Sin embargo, advierten las mismas investigadoras que no es posible generar una hipótesis explicativa al respecto, pues habría que estudiar a profundidad otras posibles hipótesis, por el momento se deja la inquietud de que los sistemas de apoyo a los estudiantes de niveles socioeconómicos están dando buenos resultados.

Variables demográficas: condiciones como la zona geográfica de procedencia, zona geográfica en la que vive el estudiante en época lectiva entre otros, son factores que eventualmente se relacionan con el rendimiento académico en forma positiva o negativa. Trabajos como el de Carrión (2002), con estudiantes universitarios en Cuba, analizaron variables demográficas, dentro de las cuales se pudo concluir que variables como la procedencia del alumno es un predictor relevante del rendimiento académico.

\subsection{Determinantes instituciona-}

les: esta categoría es definida por Carrión (2002), como componentes no personales que intervienen en el proceso educativo, donde al interactuar con los componentes personales influye en el rendimiento académico alcanzado, dentro de estos se encuentran: metodologías docentes, horarios de las distintas materias, cantidad de alumnos por profesor, dificultad de las distintas materias entre otros que seguidamente se abordarán en forma individual. Los elementos que actúan en esta categoría son de orden institucional, es decir condiciones, normas, requisitos de ingreso, requisitos entre materias, entre otros factores que rigen en la institución educativa.

Los factores institucionales tienen gran importancia en estudios sobre factores asociados al rendimiento académico desde el punto de vista de la toma de decisiones, pues se relacionan con variables que en cierta medida se pueden establecer, controlar o modificar, como, por ejemplo, los horarios de los cursos, tamaños de grupos o criterios de ingreso en carrera. (Montero y Villalobos, 2004)

Al igual que las categorías denominadas personales y sociales, los factores de índole institucional que inciden en el rendimiento académico del estudiante, puede presentar interrelaciones que se producen entre sí, y entre variables personales y sociales. La siguiente figura ilustra los factores asociados al rendimiento académico de índole institucional, agrupados en la categoría denominada determinantes institucionales.

Elección de los estudios según interés del estudiante: se refiere a la forma o vía por la cual el estudiante ingresó a la carrera, si fue su primera elección, si fue por traslado de carrera o por no haber encontrado cupo en otra carrera por ejemplo. Esta subcategoría se encuentra traslapada con los determinantes personales, pues tanto tiene que ver un asunto de orientación vocacional como con la capacidad de la Universidad en la asignación de cupos, que al final lo viene a determinar la calificación alcanzada en las pruebas de acceso y específicas de cada carrera universitaria. 
Figura N. ${ }^{\circ} 3$

Factores asociados al rendimiento académico en estudiantes universitarios

Determinantes Institucionales

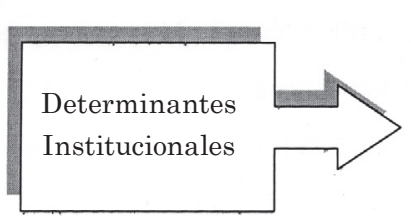

Elección de los estudios según interés del estudiante Complejidad en los estudios

Condiciones institucionales

Servicios institucionales de apoyo

Ambiente estudiantil

Relación estudiante - profesor

Pruebas específicas de ingreso a la carrera

El ajuste con lo deseado y lo real es un excelente predictor del desempeño académico universitario, son alumnos vocacionalmente satisfechos. La posibilidad de ubicarse en una carrera universitaria escogida en primera opción de interés del estudiante, también representa un indicador valioso en el rendimiento académico del estudiante universitario ${ }^{7}$. Estudios realizados por Salonava, Martínez, Bresó, Llorens, Gumbau S., Gumbau, Grau R. (2005) con estudiantes universitarios destacaron que el tipo de carrera universitaria según la satisfacción en torno a la carrera en que se encuentra; el orden de su escogencia poseen relaciones importantes en lo que a rendimiento académico se refiere.

Sobre este tema en estudio realizado por Montero y Villalobos (2004) en la Universidad de Costa Rica, no se presentó diferencia controlando el resto de variables; es decir, no se encontró diferencia en el rendimiento académico si la carrera fue o no la primera opción seleccionada por el estudiante.

Complejidad de los estudios: se refiere a la dificultad de algunas materias de las distintas carreras o áreas académicas que usualmente las universidades las clasifican basándose en estadísticas de aquellas materias con mayores índices de reprobación; la variable de este tipo fue estudiada por Rodríguez, Fita, Torrado (2004) con estudiantes universitarios y mostró relaciones importantes. La complejidad de los estudios también fue abordada por Salonava, Martínez, Bresó, Llorens, Gumbau S., Gumbau, Grau R. (2005) con estudiantes universitarios en la Universidad de Jaume I, donde se destacó la complejidad de los estudios como una variable importante en el rendimiento académico.

\section{Condiciones institucionales:} Los estudiantes también pueden ver afectado su rendimiento académico con aspectos relacionados con la universidad misma. Estudio realizado por (Salonava, Martínez, Bresó, Llorens, Gumbau S., Gumbau, Grau R. (2005) con estudiantes universitarios en la Universidad de Jaume I, indican que elementos como: condiciones de las aulas, servicios, plan de estudios y formación del profesorado, se presentan como obstaculizadores del rendimiento académico; que a su vez también pueden ser facilitadores.

Un estudio realizado por Montero y Villalobos (2004) sobre el rendimiento académico en estudiantes universitarios en la Universidad de Costa Rica, advierte sobre la importancia para la toma de decisiones, que se indicó al inicio de este 
apartado, de los factores institucionales y pedagógicos, por ser fácilmente controlados por la institución educativa. En los aspectos pedagógicos están las metodologías docentes y métodos de evaluación y en los institucionales están el número de grupo, procedimientos de ingreso a carrera y horarios de las materias.

Los factores institucionales son de gran importancia en estudios sobre factores asociados al rendimiento académico desde el punto de vista de la toma de decisiones, pues tienen que ver con variables que en cierta medida se pueden establecer, controlar o modificar, por ejemplo, los horarios de los cursos, tamaños de grupos o criterios de ingreso en carrera.

\section{Servicios institucionales de} apoyo: se refiere a todos aquellos servicios que la institución ofrece al estudiantado, principalmente según su condición económica, como lo son: sistemas de becas, servicio de préstamo de libros, asistencia médica, apoyo psicológico, entre otros.

Ambiente estudiantil: un ambiente marcado por una excesiva competitividad con los compañeros puede ser un factor tanto obstaculizador como facilitador del rendimiento académico. Se destacó la solidaridad, el compañerismo, y el apoyo social como importantes elementos que inciden positivamente $^{8}$.

Relaciones estudiante profesor: las expectativas que el estudiante tiene sobre las relaciones con sus profesores y con sus compañeros de clase son factores importantes que intervienen en los resultados académicos. Al respecto Castejón \& Pérez (1998) hacen referencia a que el estudiante desea encontrar en el profesor tanto una relación afectiva, como didáctica y que ello tiene repercusiones en el rendimiento académico.

Pruebas especificas: se refiere a aquellas carreras universitarias que establecen además de las pruebas de admisión a la universidad, pruebas específicas de aptitud con la carrera que el estudiante desea cursar. Carrión (2002) les atribuye a las pruebas que de una $u$ otra forma determinan el ingreso al estudiante un valor importante en materia de rendimiento académico. Esta pruebas principalmente se asocian con los cupos disponibles, como un criterio más de selección.

Después de haber realizado esta sistematización en torno a factores asociados al rendimiento académico, es importante advertir que, dada la complejidad del tema, es posible que valiosos indicadores se han dejado por fuera y, que en las relaciones que se suelen dar entre los distintos factores por más relaciones que parezcan tener, no es posible hablar de una generalización, pues los resultados podrían ser muy diferentes según el conjunto de las relaciones causales, las poblaciones que se estudien y sus contextos. Por lo general estudios orientados hacia el rendimiento académico, utilizan, en su metodología modelos de regresión múltiple y se encontró en ellos facilidades para analizar las relaciones experimentadas entre la interacción de las variables involucradas, a fin de tratar de llegar a determinar cuáles son los factores que con mayor peso predictivo permitan una mejor explicación con el objeto de estudio.

Estudios enfocados hacia el rendimiento académico en la educación superior poseen un alto valor para comprender en mayor medida el complejo mundo de la calidad de la educación superior pública, cuestionada por distintos sectores y desde ópticas diferentes.

\section{Rendimiento académico y calidad de la educación superior}

Tal y como se mencionó al inicio de este artículo, conocer con mayor precisión sobre el rendimiento académico de los estudiantes universitarios es un indicador clave 
en la calidad; así lo deja ver la Comisión on Higher Education MSA, 1995, citado por Rodríguez, Fita, Torrado (2004, p. 394):

El rendimiento académico de los estudiantes es un componente clave para determinar si una institución está alcanzando sus objetivos educativos. De aquí, que sea esencial la existencia de un programa de evaluación para documentar el rendimiento académico de los estudiantes.

Los resultados del sistema universitario se miden principalmente por el éxito académico alcanzado en sus alumnos, la idoneidad de sus graduados, sus tiempos de duración, su inserción laboral entre otros rubros. Esto no quiere decir que no haya otros indicadores valiosos como los programas de investigación, la proyección social, por ejemplo; lo que sí está claro es que la proyección social de las universidades, así como sus programas de investigación, nacen con los aportes de sus profesionales; he ahí la importancia de monitorear sus resultados académicos, conociendo para ello los factores que mayor incidencia representan en los resultados académicos de los estudiantes y diseñar estrategias de intervención en la medida de lo posible, desde donde a la universidad le corresponde. Muchos factores pueden ser manipulados desde la responsabilidad de la universidad, pero también es cierto que hay otros que no son de la competencia universitaria.

No obstante, la calidad de la educación superior pública, al cuestionarse sus resultados en cuanto a la eficacia e eficiencia, en una relación costo-beneficio estatal, donde intervienen recursos limitados del Estado, se supone un uso racional de sus recursos, con un máximo aprovechamiento y una mayor incidencia social, donde la orientación fundamental es que los estudiantes admitidos logren su titulación en los tiempos estipulados, aumentado así la inversión social.

Tomando en cuenta la limitación de los recursos estatales para la educación superior pública, principalmente en los últimos años, son imprescindibles investigaciones en el campo del rendimiento académico, que además de permitir una aproximación a la realidad estudiantil desde esta óptica, represente para las instituciones un insumo valioso para la toma de decisiones y para el Estado un indicador de inversión; no contar con este tipo de información sería debilitar la sostenibilidad de las decisiones, asumiendo el costo de las repercusiones sociales que esto conllevaría, y más aún cuando la relación entre la cantidad de estudiantes que acceden a la universidad y los que logran titularse en tiempos racionales, es frecuentemente usado en las universidades como un importante indicador de calidad y por el sector estatal como elementos decisorios en lo que a inversión se refiere. (Rodríguez, Fita, Torrado, 2004)

La discusión en torno a la calidad de la educación superior es ampliamente controversial por su misma naturaleza conceptual, no hay un criterio consensuado; en ella intervienen múltiples factores de distinta complejidad, y el rendimiento académico de sus estudiantes es solo una dimensión de su análisis y quizás la más importante, pues el fin último de las universidades públicas es aportar con la formación profesional idónea lo que la sociedad ha invertido en ellas, y así buscar las transformaciones que la sociedad requiere. El tema del rendimiento académico parece compartir un nivel de dificultad conceptual similar al de la calidad de la educación, por intervenir, en ambos, ambiguos y variados factores en diferentes circunstancias y momentos ${ }^{9}$.

En lo que sí parece haber consenso es en que ambos temas, tanto el rendimiento académico como la calidad educativa, conllevan implícito una posición integral en su abordaje, tal y como lo expone Dias Sobrinho, 1995, citado por Lamarra (2004), al referirse a la conceptualización de la calidad educativa en la que hace ver que es una construcción social, que varía según los intereses de quien la mire y que lleva 
articulada las características que la sociedad le ha marcado.

La búsqueda de la calidad educativa en el sector universitario se ha asumido entre los distintos debates, como un bien deseado por distintos sectores de la sociedad, por el que luchan las universidades desde diferentes ámbitos, obviamente con mayores cuestionamientos en el sector público por la inversión estatal que hay de por medio, tomando en cuenta que en el actual marco social, caracterizado por un mundo globalizado, estos recursos son cada día más limitados. La búsqueda de esta calidad implica una revisión integral de la universidad y los estudios en torno al rendimiento académico de sus alumnos vienen a ser un insumo más importante de lo que muchas veces se le atribuye, puesto que permite conocer aquellos elementos que obstaculizan y que facilitan el desempeño estudiantil y por ende permite monitorear la utilización de los recursos estatales y su impacto social. También son una forma de profundizar en el tema tan complejo de las desigualdades sociales, pues Marchesi (2000), considera que las desigualdades son demostradas en los análisis de rendimiento académico por clases sociales, al considerar que es previsible encontrar diferencias entre los estudiantes debidas a su origen social. Por su lado, Camps (1997), refiriéndose al rendimiento académico, considera que los malos resultados académicos son un muestra incuestionable de que la equidad educativa no se consigue; sin embargo, este tema por la ambigüedad que trae implícita, merece un tratamiento profundo, que para efectos de este artículo se deja ver la importancia de ambas variables: rendimiento académico y equidad, dejando claro que el tema de diferencias sociales no es una responsabilidad exclusiva del sistema universitario.

Un correcto análisis de la calidad educativa, que incluya la temática en torno al rendimiento académico de los estudiantes, es de gran utilidad en procesos de toma de decisiones en aras de un sistema educativo más justo.

Como se muestra en la Figura No. 4 la interacción analítica y causal entre factores asociados al rendimiento académico como los determinantes personales, sociales e institucionales, repercuten en la calidad educativa que se aspira.

Figura N. ${ }^{\circ} 4$

Interacción entre factores asociados al rendimiento académico

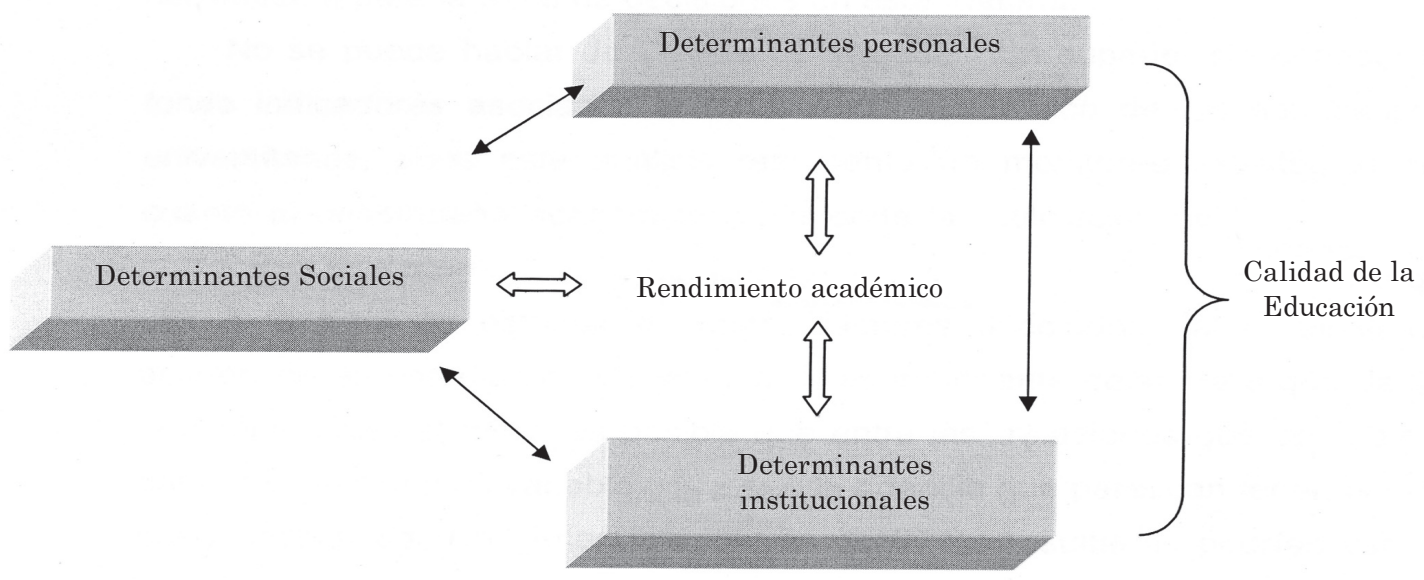


Finalmente, es de interés considerar que el análisis del rendimiento académico de los estudiantes universitarios mediante la investigación, constituye un factor imprescindible en los debates en torno a la búsqueda de la calidad de la educación superior, es un indicador fundamental que permite desde esta óptica una aproximación a la realidad educativa; ofrece, además, sólida información para la toma de decisiones en educación superior pública mediante la identificación de criterios de desempeño estudiantil, que permitan sustentar la relación costo e inversión estatal.

No obstante, el rendimiento académico es el resultado de la suma de diferentes y complejos factores que actúan en la persona que aprende, donde entran en juego interacciones de determinantes personales, sociales e institucionales en las que se desenvuelve el estudiante, cuyos resultados se les atribuye un valor mediante las calificaciones que se le otorgan al estudiante, que podrían dar como resultado: abandono, retraso y éxito académico, situación que finalmente conduce a conocer la relación entre lo que se aprende y lo que se logra desde el punto de vista del aprendizaje.

El conocer los posibles factores que mayormente inciden en el rendimiento académico en estudiantes universitarios permitiría al menos, entre otros, predecir posibles resultados académicos y poder hacer un análisis sobre su incidencia en la calidad educativa que se espera y ser una herramienta para la toma de decisiones en esta materia.

No se puede hablar de calidad de la educación superior sin conocer a fondo indicadores asociados al rendimiento académico de los estudiantes universitarios, pues este análisis representa un monitoreo estratégico en cuanto al desempeño académico y por ende la utilización de los recursos que el Estado invierte.

A la hora de estudiar diferentes factores asociados al rendimiento académico en estudiantes universitarios, es importante dejar claro que dada la complejidad del tema, es posible que entre las relaciones que se suelen dar entre las distintas variables por más incidencia que parezcan tener, no se puede hablar de una generalización; es decir, los resultados podrían variar según el conjunto de interacciones entre sí, tan distintas como poblaciones que se estudien y sus contextos.

La discusión en torno a la calidad de la educación superior es controversial por su misma naturaleza conceptual, no hay un criterio consensuado; en ella intervienen múltiples factores de importancia, y el rendimiento académico de sus estudiantes es solo una dimensión de su análisis y posiblemente la más valiosa, tomando en cuenta que dentro de los propósitos de las universidades públicas está el aportar las trasformaciones que la sociedad requiere mediante el aporte de profesionales idóneos, lo cual supone un tiempo racional en su formación.

El estudio del rendimiento académico comparte con la calidad de la educación su complejidad conceptual, al intervenir en ambos distintos factores que según sus enfoques de estudio y contextos desprenden disímiles resultados.

\section{Notas}

1. Díaz, M., Peio, A., Arias. J., Escudero, T., Rodríguez, S., Vidal, G. J. (2002).

2. Salonava, M., Cifre, Eva, Grau, R., Martínez Martínez, Isabel, 2005).

3. Valle Arias, A., González Cabanach, R., Núñez Pérez, J., Martínez Rodríguez, S., Pineñor Aguin, I., 1999).

4. Mayer, 1992 citado por Valle Arias, A., González Cabanach, R., Núñez Pérez, J., Martínez Rodríguez, S., Pineñor Aguin, I., 1999).

5. Valle Arias, A., González Cabanach, R., Núñez Pérez, J., Martínez Rodríguez, S., Pineñor Aguin, I., 1999).

6. Díaz, M., Peio, A., Arias. J., Escudero, T., Rodríguez, S., Vidal, G. J., [2002] y Rodríguez, 
S., Fita, S., Torrado, M. (2004); Toca, M.T., Tourón, J. , [1989]).

7. Díaz, M., Peio, A., Arias. J., Escudero, T., Rodríguez, S., Vidal, G. J., (2002); Rodríguez, S., Fita, S., Torrado, M. (2004).

8. Salonava Soria, M., Martínez Martínez, Isabel M., Bresó, Esteve, E., Llorens Gumbau, S., Gumbau Grau, R., 2005).

9. Morillejo, E., Rebolloso, P. E., Pozo, M. C., Baltasar, F. (1999); Seibold, J. R. (2000).

\section{Referencias bibliográficas}

Beck, Urlich. (1999) ¿Qué es la globalización? Falacias del globalismo, respuestas a la globalización. Barcelona: Piadós.

Camps, Victoria. (1997). La igualdad en educación. En: Intervención Psicosocial, 1(6), 1-5.

Carrión Pérez, Evangelina. (2002, EneroMarzo). Validación de características al ingreso como predictores del rendimiento académico en la carrera de medicina. Revista Cubana de Educación Medica Superior, 1(16), Artículo 1. Extraído el 20 de enero, 2007 de http://scielo.sld.cu/scielo.php? script $=$ sci_arttext\&pid $=$ S0864-2141 2002000100001\&lng=es\&nrm=iso

Castejón, C., Pérez, S. (1998). Un modelo causal-explicativo sobre la influencia de las variables psicosociales en el rendimiento académico. En: Revista Bordon. Sociedad Española de Pedagogía. 2(50), 170-184.

Cohen, Ernesto. (2002). Educación, eficiencia y equidad: una difícil convivencia. En: Revista Iberoamericana CSIC. 30, Setiembre-Diciembre, 105-124.

Díaz, M., Peio, A., Arias, J., Escudero, T., Rodríguez, S., Vidal, G. J.
(2002). Evaluación del Rendimiento Académico en la Enseñanza Superior. Comparación de resultados entre alumnos procedentes de la LOGSE y del COU. En: Revista de Investigación Educativa, 2(20), 357-383.

Fernández Santamaría, M. del Rosario. (2000, Enero-Abril). Calidad y equidad educativas. En: Revista Iberoamericana de Educación. (OEI), 22, Artículo 10. Extraído el 31 de enero, 2007 de http://www.rieoei.org/ rie22a10.htm

González, F. A. (1996). Comprensión lectora y rendimiento académico. En: Revista Gallega de Psicopedagogía, 13(9), 209-221.

Lamarra Fernández, Norberto. (2004). Hacia la convergencia de los sistemas de educación superior en América Latina. En: Revista Iberoamericana de Educación OEI, 35, Mayo-Agosto, 1-14.

Marchesi, Álvaro. (2000). Un sistema de indicadores de desigualdad educativa. En: Revista Iberoamericana de Educación, 23, Mayo-Agosto, 1-22.

Montero Rojas, Eilena, Villalobos Palma, Jeannette. (2004). Factores institucionales, pedagógicos, psicosociales y sociodemográficos asociados al rendimiento académico y a la repetición estudiantil en la Universidad de Costa Rica. Instituto de Investigaciones Psicológicas, Universidad de Costa Rica.

Morillejo, E., Rebolloso, P. E., Pozo, M. C., Baltasar, F. (1999). Evaluación y calidad en la educación superior. En: Papeles del Psicólogo. PSICODOC, 74, Noviembre, 1-6.

Oliver, Juan C. (2000). Multinivel regresión models: apllications in Scholl 
psychology. En: CSI Psicothema, 3(12), 487-494.

Pelegrina, Saniago García, Linares M. C, Casanova, Pedro F. (2002). Parenting styles and adolescents' academic perfomance. En: Infancia y aprendizaje. (Revista electrónica USAL), 25(2), 147-168.

Pérez-Luño, A., Ramón Jerónimo, J., Sánchez Vázquez, J. (2000). Análisis exploratorio de las variables que condicionan el rendimiento académico. Sevilla, España: Universidad Pablo de Olavide.

Rodríguez, S., Fita, S., Torrado, M. (2004). El rendimiento académico en la transición secundaria-universidad. En: Revista de Educación. Temas actuales de enseñanza, 334, Mayo-Agosto.

Salonava Soria, M., Martínez Martínez, Isabel M., Bresó, Esteve E., Llorens Gumbau, S., Gumbau Grau, R. (2005). Bienestar Psicológico en estudiantes universitarios: facilitadores y obstaculizadores del desempeño académico. En: CSIC. Anales de Psicología, 1(21, junio), 170-180.

Salonava, M., Cifre, Eva, Grau, R., Martínez Martínez, Isabel (2005). Antecedentes de la autoeficacia en profesores y estudiantes universitarios: un modelo causal. En: Revista de Psicología del Trabajo y de las Organizaciones. 1-2(21), 159-176.

Seibold, J. R. (2000). ¿Equidad en la Educación? Reflexiones sobre un nuevo concepto de calidad educativa que integre valores y equidad educativa. En: CSIC Revista Iberoamericana de Educación, 23, Mayo-Agosto, 1-12.

Soria, Oscar. (1993). Descentralización educativa. El dilema entre saber, poder y querer ¿Una nueva Universidad para el s. XXI. En: Revista Iberoamericana de Educación. OEA, 3, SetiembreDiciembre, 1-10.

Toca, M. T., Tourón, J. (1989). Factores del rendimiento académico en los estudios de arquitectura. En: Revista de Investigación Educativa. 14(7), 31-45.

Valle Arias, A.; González Cabanach, R.; Núñez Pérez, J.; Martínez Rodríguez, S; Pineñor Aguin, I. (1999). Un modelo causal sobre los determinantes cognitivo-motivacionales del rendimiento académico. En: Revista de Psicología General Aplicada. 52(4), 499-519.

Vélez Van, M. A., Roa, N. C. (2005). Factors associated with academic perfomance in medical students. En: PSIC. Educación Médica. 2(8), 1-10. 